\title{
A numerical and experimental study of a thin, high cambered airfoil at a low Reynolds number
}

\author{
A. Auletta ${ }^{1}$, F. De Gregorio ${ }^{1}$, D. Guida ${ }^{1}$, M. Lupo $^{2} \&$ M. Marrazzo ${ }^{1}$ \\ ${ }^{1}$ Italian Aerospace Research Centre (CIRA), Italy \\ ${ }^{2}$ University of Rome "La Sapienza”, Italy
}

\begin{abstract}
In the framework of the development of new aerodynamic design concepts, a joint numerical/experimental analysis has been performed on a $2 \mathrm{D}$ thin, high cambered airfoil at a low Reynolds number. The experimental investigation has been performed by means of the Pressure Sensitive Paint Technique (PSP) and Particle Image Velocimetry (PIV). These techniques have been selected for their characteristics of low-intrusivity and high spatial resolution. Details about the experimental set-up and results shall be reported. The experimental investigation has been intended to obtain detailed information about the pressure field and to detect flow characteristics on a non-conventional airfoil. Experimental data have been compared with numerical results obtained by FLUENT commercial code.

Results will be presented in terms of experimental and numerical velocity fields around the model and in terms of lift characteristics.
\end{abstract}

Keywords: PIV, PSP, endurance, non-planar wing, aerodynamic loads.

\section{Introduction}

In the framework of the Unmanned Aerial Vehicle (UAV) Project, which is aimed at designing a High Altitude Long Endurance (HALE) unmanned vehicle, some configurations have been tested. In more detail, a prerogative of HALE vehicles has the possibility of operating at high altitudes ranging between 20000-30000 metres, with an endurance of at least 48 hours. One of the considered configurations has been characterized by an inflatable leading edge so that an airfoil characterized by high curvature and small thickness has been produced, similar to a modern directional parachute (Figure 1). A wide 
numerical and experimental test campaign has been performed in order to analyse the aerodynamic behaviour of this configuration and to confirm the preliminary analysis carried out during the design phase.

\section{Experimental set-up}

\subsection{Wind tunnel}

Tests have been carried out in the Calibration Tunnel 1 (CT-1). CT-1 is an Eiffel type open circuit wind tunnel, with the following main characteristics:

○ velocity range: $5-55 \mathrm{~m} / \mathrm{s}$

○ nozzle contraction rate: $16: 1$

- maximum value of turbulence level at $50 \mathrm{~m} / \mathrm{s}$ flow speed: $<0.1 \%$

$\circ$ test section size $(\mathrm{L} \times \mathrm{W} \times \mathrm{H}): 600 \times 305 \times 305 \mathrm{~mm}^{3}$.

The test section allows in the region of $100 \%$ optical access. It is equipped with a motorized turntable equipped with an angular decoder providing the geometric incidence angle of the model to the data acquisition system.

\subsection{Test article}

The investigated profile is thin like a modern directional parachute. It was designed in the framework of the project Non-Planar Wing (NPW) by CIRA researchers G. Andreutti and D. Quagliarella. A sketch of the geometry is shown in Figure 1. The main dimensions of the test model are as follows:

o span: $302 \mathrm{~mm}$

○ chord: $100 \mathrm{~mm}$

o maximum thickness: $6.26 \mathrm{~mm}$.

The test article is equipped with fifteen pressure taps, nine located on the upper surface and six located on the lower surface. The location of the pressure taps has been concentrated on the leading edge of the model in order to integrate the data coming from the PSP affected for some incidence angles by a lack of information.

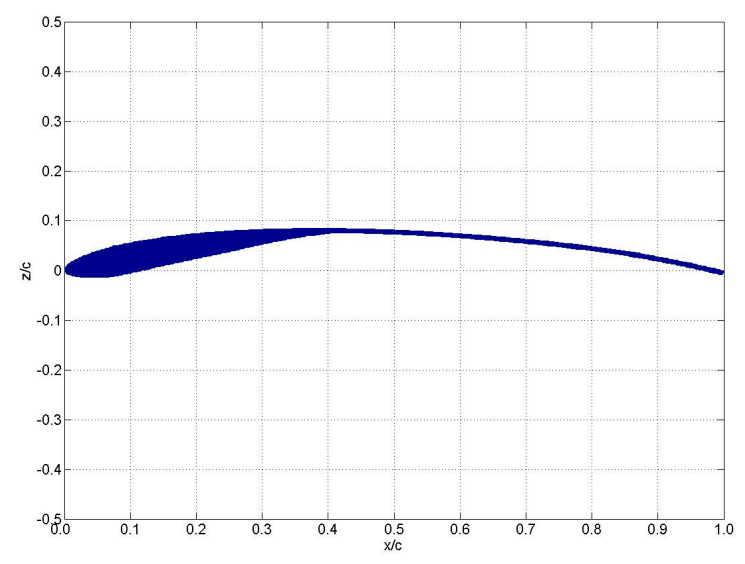

Figure 1: Investigated airfoil. 


\subsection{PIV system}

The laser system is composed of two Nd-Yag resonator heads providing a laser beam of about $300 \mathrm{~mJ}$ each at $532 \mathrm{~nm}$. The light sheet is delivered in the measurement area by means of two mechanical systems composed of a mechanical arm equipped with seven mirrors and which provides six freedom degrees. Measurements have been performed on the symmetry vertical plane of the test section in order to minimize possible wall effects and assure the bi-dimensionality of the phenomena.

A large seeding generator, equipped with twenty Laskin nozzles, has been used. A cyclone has been mounted in order to filter large droplets and avoid environmental contamination. DEHS oil has been adopted as the seeding material. In order to obtain a uniform concentration of particles the full wind tunnel room has been seeded.

Two CCD cameras equipped with $28 \mathrm{~mm}$ Zeiss lenses and mounted on a 2D linear traversing system have been used for measuring the flow field around the model and in the wake regions.

\subsection{PSP system}

The paint employed was the single component UniFIB, manufactured by ISSI. Paint luminescence was induced by three blue LED lamps (with a spike at 405 $\mathrm{nm}$ ) equipped with a $10 \mathrm{deg}$. angle diffuser and supported by a X95 frame. Long-term stability is about $1.0 \%$ after 5 minutes, and $0.1 \%$ after 20 minutes of operation.

A Princeton Instruments 16-bit CCD back illuminated camera (model Pixis 1024B) equipped with a $50 \mathrm{~mm}$, f1.8D Nikkor lens has been used for the images' acquisition. The camera is characterized by a $1024 \times 1024$ pixel resolution, a quantum efficiency of about $95 \%$ in the visible range and a full well capacity of $240 \mathrm{ke}^{-}$. The camera position has been chosen in order to look at the entire model under the entire test campaign conditions.

\section{Numerical flow solver algorithms}

Numerical simulations have been carried out by means of Drela's MSES code and FLUENT commercial code.

MSES [3] is based on a finite-volume discretization of the Euler equations on a streamlined grid. Also in this case a compressible formulation has been used. The MSES code computes the viscous region by using an integral boundary layer formulation based on a multi-layer velocity profile representation. The inviscid and viscous regions are coupled by using the displacement thickness theory.

FLUENT $^{\mathrm{TM}}$ solves the Navier-Stokes equations on hybrid grids by means of the Finite Volume method. A second order upwind scheme has been adopted for the spatial discretization whereas an incompressible formulation was chosen as the governing mathematical model. For such a flow model the continuity is 
enforced by means of a Poisson equation for the pressure. The pressure-velocity coupling was ensured by means of the SIMPLEC algorithm and by using the pressure-staggering approach "PRESTO!". In some cases convergence problems occurred, therefore a collocated second order pressure interpolation scheme has been used instead. Moreover, due to the isothermal flow assumption, the energy equation was disregarded. In all cases the so called segregated implicit solution procedure was used, where each single flow equation is solved sequentially. Furthermore, the following boundary conditions types have been used in the computations.

The so called "velocity inlet" boundary condition was used to prescribe the velocity profile at the left boundary of the computational domain.

The classical no-slip boundary condition has been adopted on the lower side of the computational domain.

The "outflow" boundary condition has been adopted on both the upper and right sides of the computational domain. In such a case the normal derivative of all the flow variables is set to zero, except for pressure.

Finally, a RANS approach has been used in order to include turbulence effects on the flow solution. Further information can be found in [4].

\section{Procedure for data reduction}

With regards to the numerical analysis, an initial investigation by means of MSES has been carried out in order to get a preliminary estimation of the aerodynamic characteristics on the project conditions (low Reynolds number) and to have information about the transition location. This latter can have a great influence on the aerodynamic characteristics because of the airfoil's large curvature and low thickness. This information has been used as a starting point for the CFD analysis on the flow field carried out through FLUENT commercial code.

With regard to the experimental analysis, velocity profiles have been evaluated using two PIV cameras aligned vertically (one for the upper part of the test section, one for the lower part), scanning the entire flow region around and behind the model at different instants. The mean fields obtained for each region, partially overlapped with others, have been merged together to allow the necessary integration process for the aerodynamic coefficients evaluation.

The lift coefficient has been calculated applying the Kutta-Joukowski theorem on a circulation line surrounding the airfoil in the measurement plane. The drag coefficient, on the other side, has been evaluated using the momentum balance equation. All the results have been corrected taking into account the wall effects and compared with the numerical ones for different angles of attack.

The integration of pressure distributions obtained by PSP provided another criterion for an experimental measurement of the lift coefficient, as well as for the moment coefficient. The application of the PSP technique has been forced by the reduced dimensions of the test article that would not allow the insertion of a reasonable number of pressure taps, especially in the aft part of the model. 


\section{Results and discussion}

In the scenario of experimental measurement technique for aerodynamics, PIV provides a unique tool in terms of data accuracy, test productivity and, at the same time, an understanding of the phenomena. PIV data give a clear general view of the flow field behaviour. Velocity field colormaps, around the model and in the wake region, for $\alpha=-4^{\circ}, 0^{\circ}$ and $+4^{\circ}$ are reported in Figures 2-4 Plots give an insight into the aerodynamic behaviour at different angles of attack, showing a flow separation on the lower surface for the negative angle confirmed by the large wake left behind. Partial separation occurs at $\alpha=0^{\circ}$. For the positive angle of attack shown, the flow remains attached on the lower side of the model. The PIV analysis has shown that on the upper surface a trailing edge flow separation starts from about $11^{\circ}$. The flow field at $15^{\circ} \mathrm{AoA}$ is reported in Figure 5.

Figure 5 shows the velocity profile along the height of the test section ( $\mathrm{Z}$ direction) two chords downstream from the model trailing edge for the highest investigated angles of attack $\left(\alpha=11^{\circ}-15^{\circ}\right)$. It can be seen that the wake at $11^{\circ}$ and $12^{\circ}$ shows quite the same extent whereas the momentum loss increases slightly starting from $14^{\circ}$. This is also highlighted by a soft stall starting from $12^{\circ} \mathrm{AoA}$ as presented in Figure 8 .

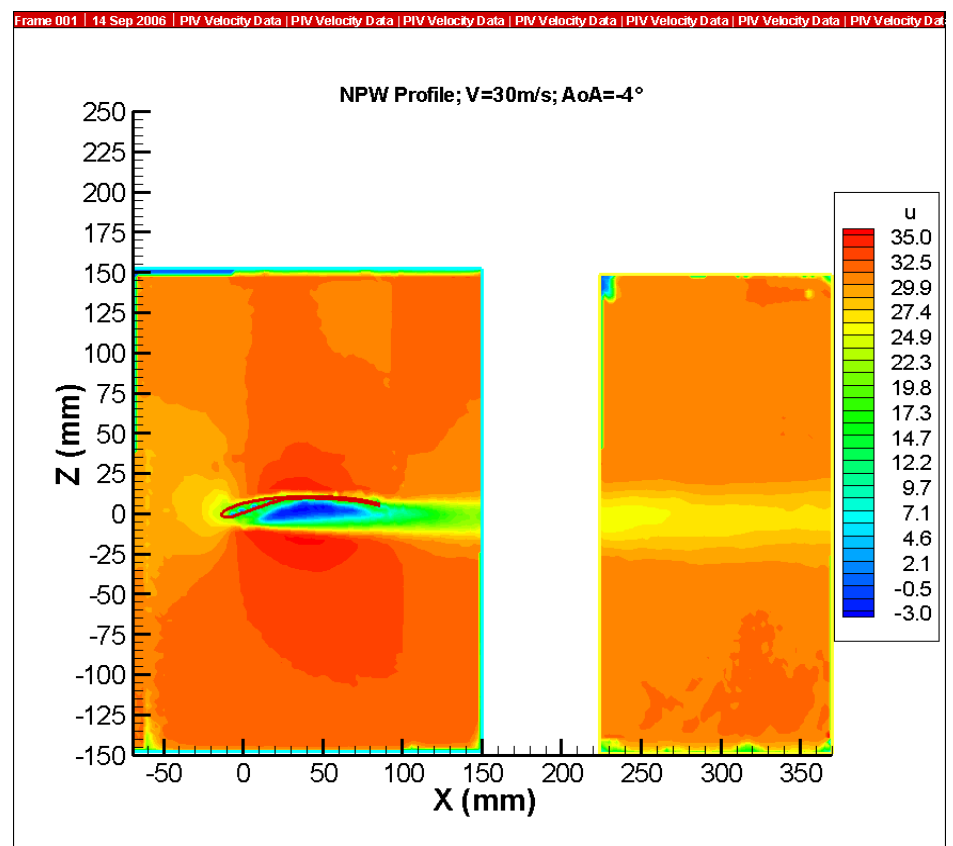

Figure 2: $\quad$ PIV flow field at $-4^{\circ}$ AoA. 
186 Advances in Fluid Mechanics VII

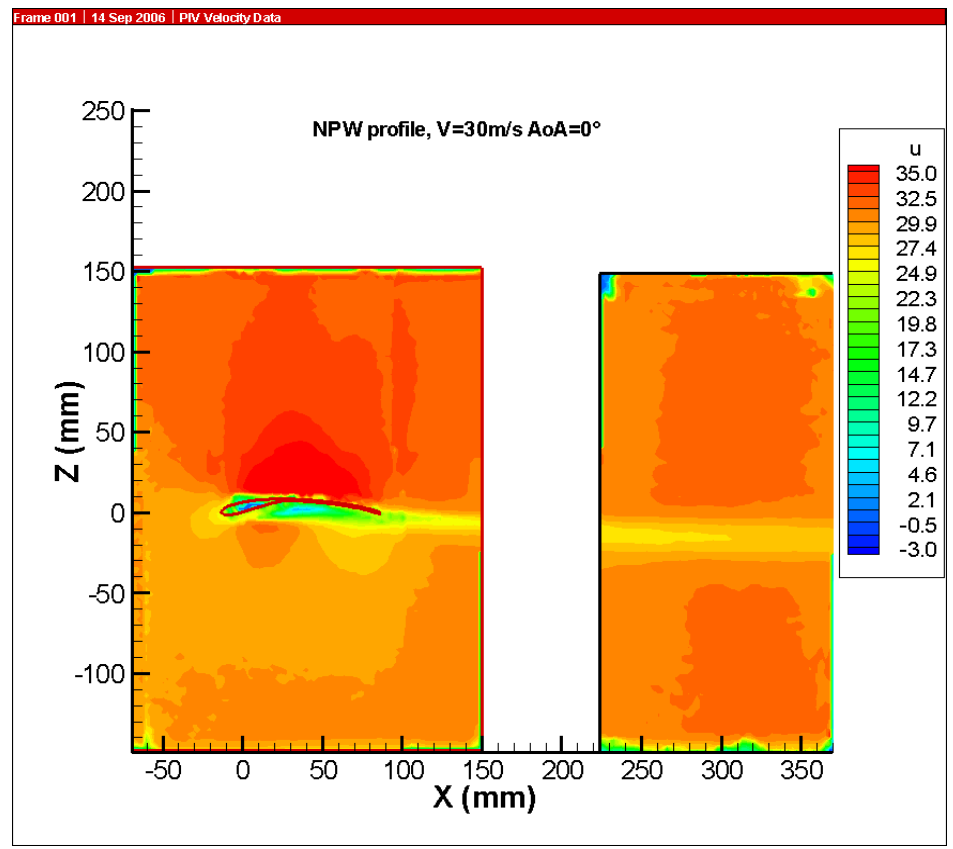

Figure 3: $\quad$ PIV flow field at $0^{\circ}$ AoA.

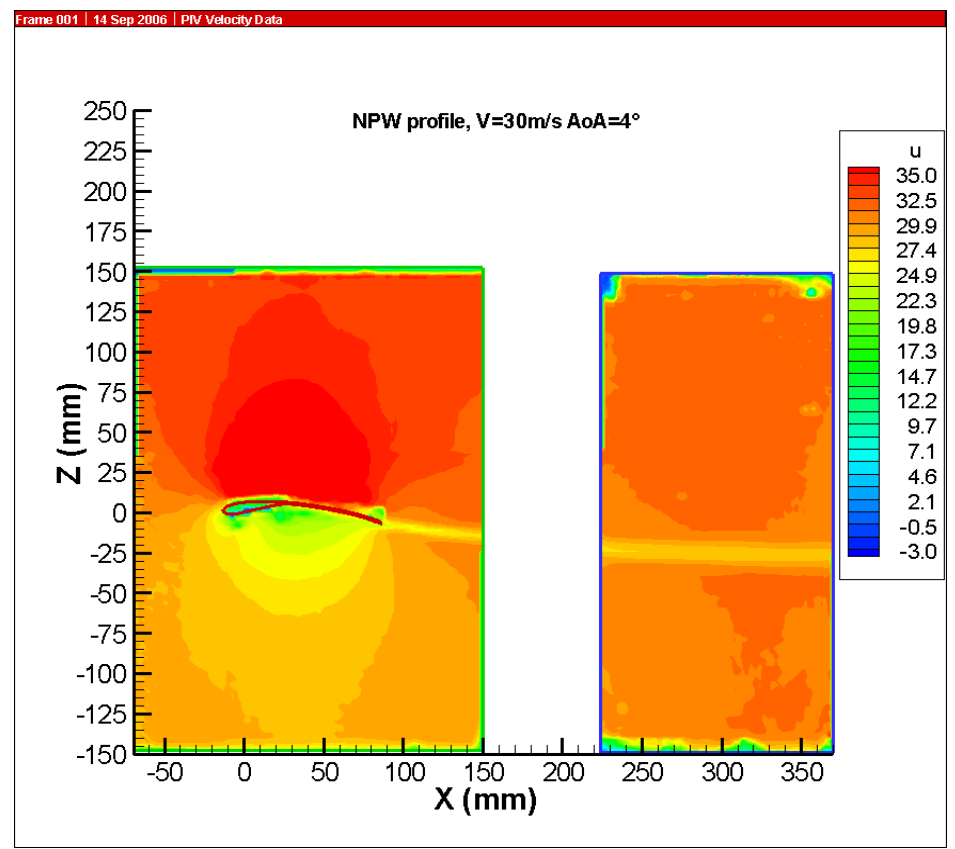

Figure 4: $\quad$ PIV flow field at $4^{\circ}$ AoA. 


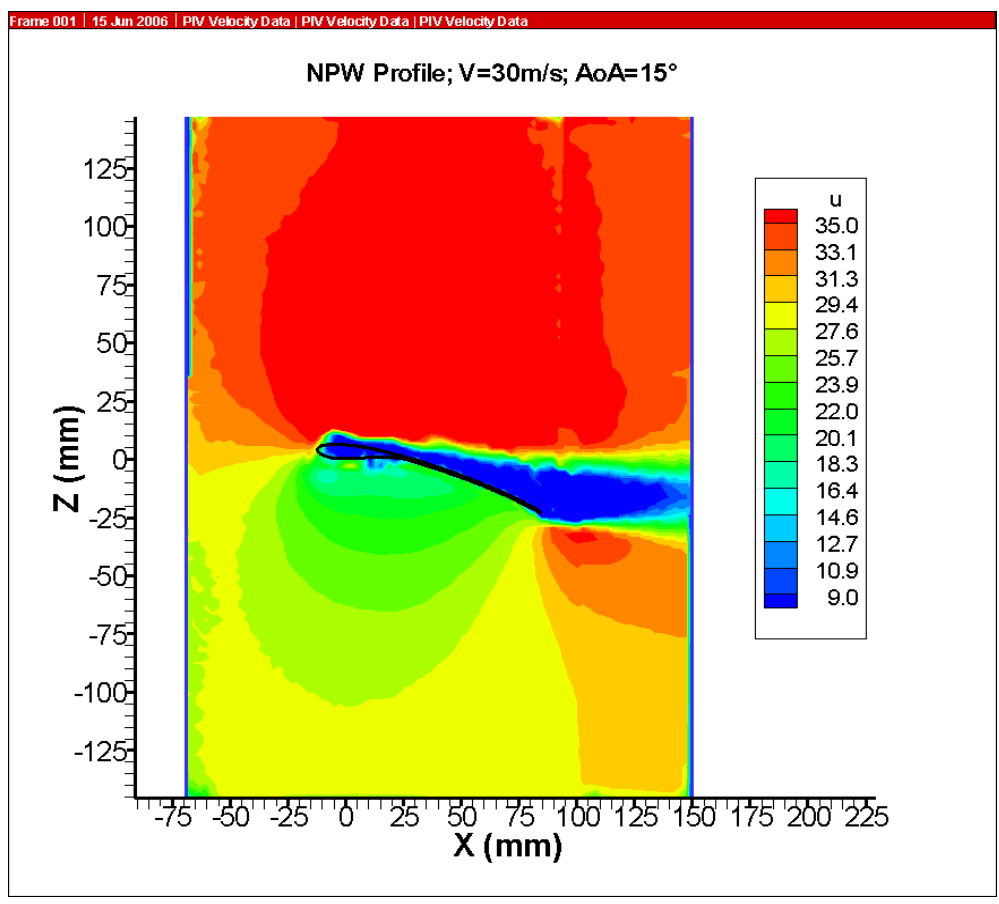

Figure 5: $\quad$ PIV flow field at $15^{\circ}$ AoA.

Numerical simulations have highlighted that laminar-turbulent transition plays an important role on the flow field. In Figure 7, the flow field obtained considering a fully turbulent flow is compared with the free transition flow field at $0^{\circ}$ AoA. It can be seen that flow characteristics are dramatically affected by transition position. The field predicted by a transition free criterion is confirmed by the experimental flow field shown in Figure 3, where a wide region of separated flow is evident in the lower part of the airfoil. The impact of the numerical algorithm chosen (fully turbulent or transition free) is also evident looking at the numerical/experimental comparison as reported in Figure 8 in terms of lift coefficient distribution. PIV and PSP integrated data are compared with results of RANS calculations for both fully turbulent and transition free conditions. It is noticeable that the experimental lift curves are close to each other whereas a sensible divergence is shown by the CFD fully turbulent curve. On the contrary, transition free simulations seem to provide lift values coherent with the experimental measurements. This suggests that the laminar-to-turbulent transition sensibly affects the flow field so that, the hypothesis of a fully turbulent flow on the entire model surface, eliminating the possibility of large separated regions, implies a wrong evaluation of normal strains and, in turn, of the lift coefficient.

A comparison of numerical and experimental results in terms of pressure coefficients is reported in Figure 9 for different angles of attack. Excluding the very critical case at $\alpha=0^{\circ}$, a good agreement is noticeable, although it can be 
seen that experimental (PSP) pressure profiles are not closed on the model trailing edge. This is probably due to an inaccurate measurement in the last $5-10 \%$ of chord on the lower surface (a possible reason can be paint degradation or imperfection in the paint application due to the high curvature). In fact, pressure distribution on the lower surface, very close to the trailing edge, seems to show a smaller pressure recovery than the expected one, especially at low angles of attack.

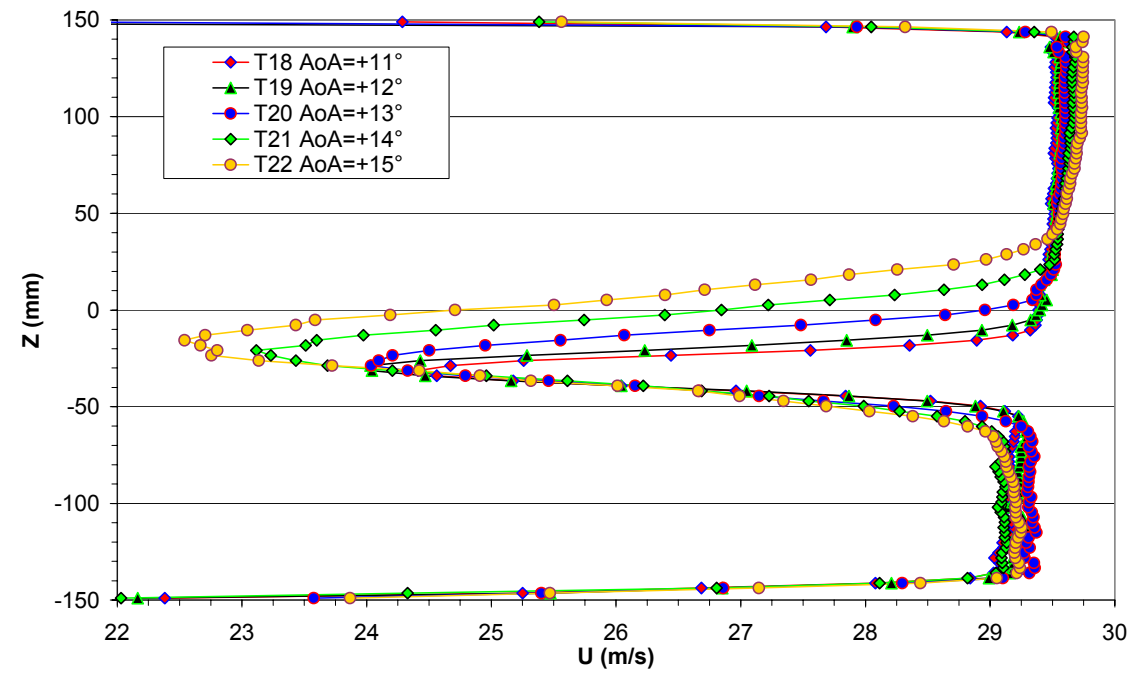

Figure 6: Wake velocity profiles (AoA between $11^{\circ}$ and $15^{\circ}$ ).

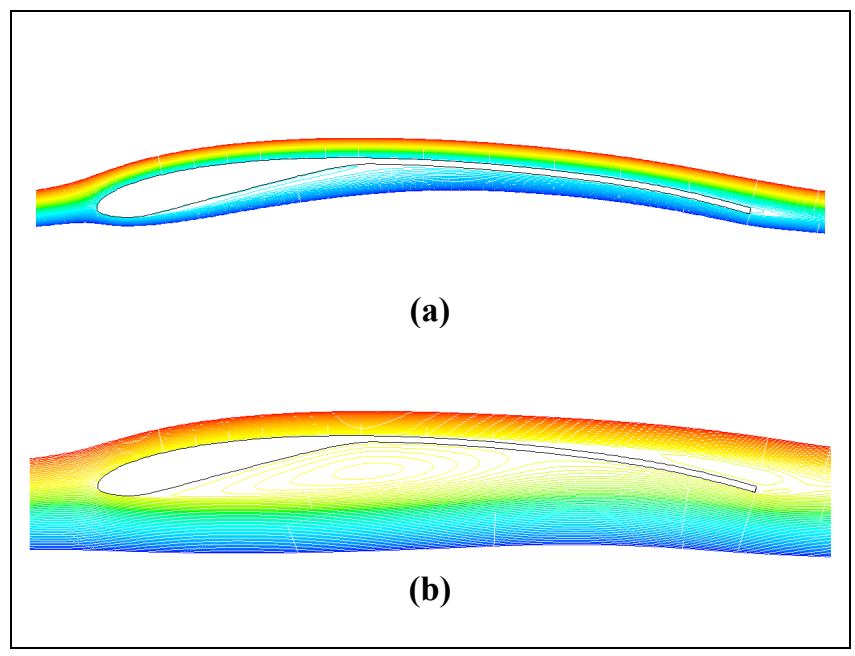

Figure 7: $\quad$ Numerical streamlines at $0^{\circ}$ AoA (a: fully turbulent, b: transition free). 


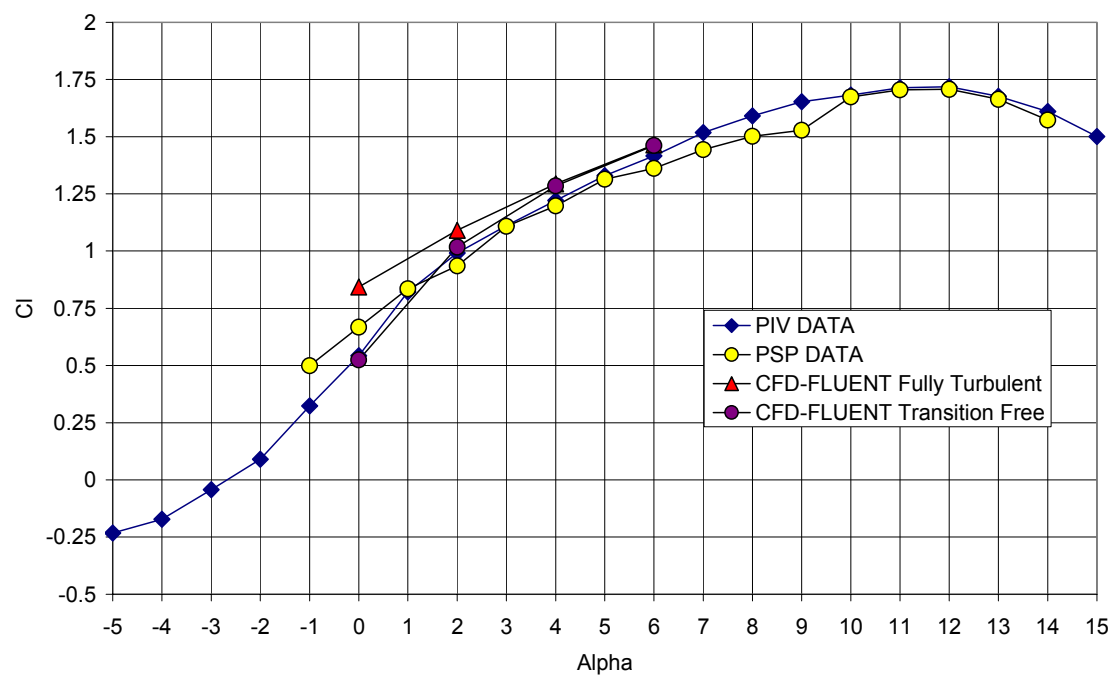

Figure 8: Comparison between numerical and experimental lift coefficient.
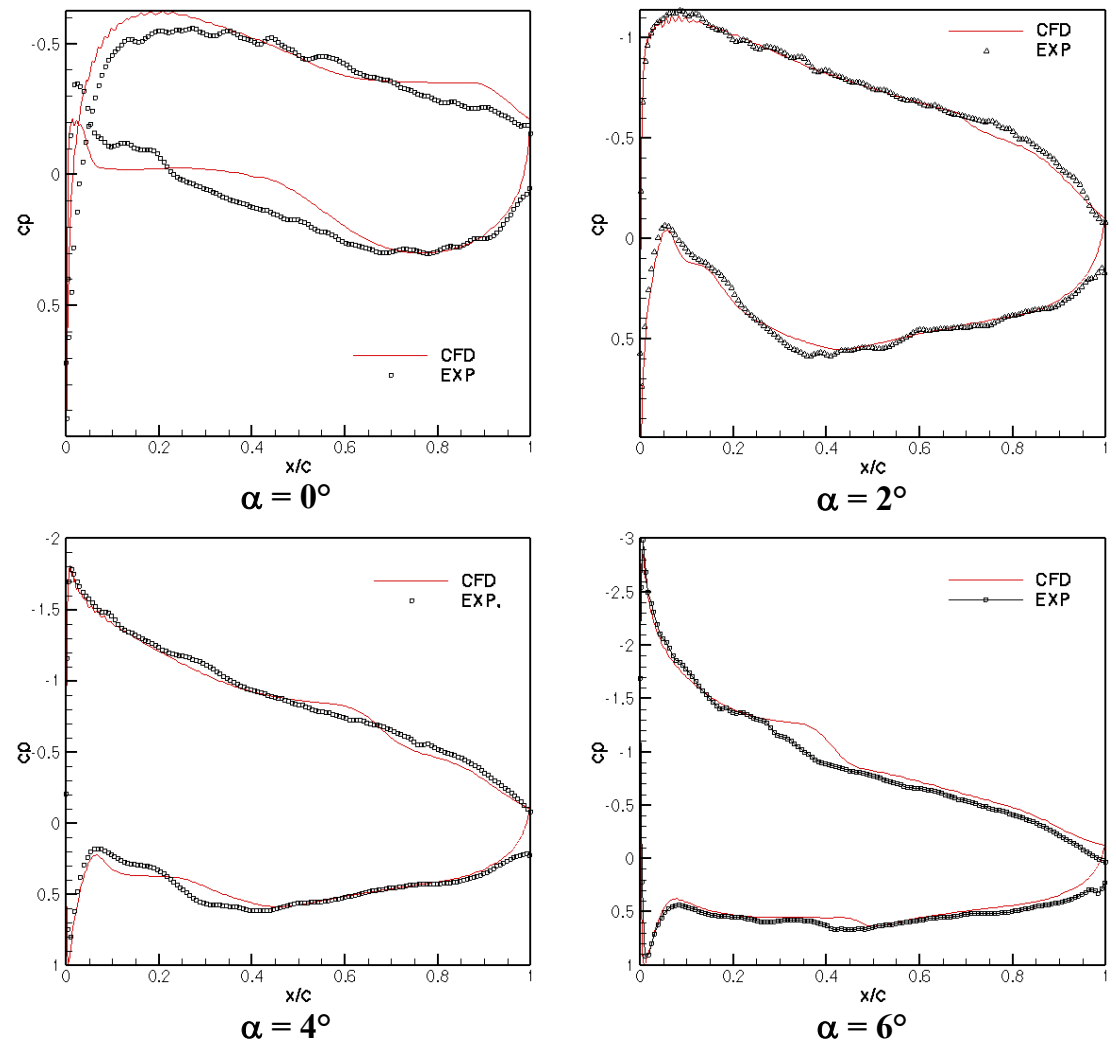

Figure 9: Comparison between CFD and PSP Cp distributions. 


\section{Conclusions}

In the framework of the Non-Planar Wing Project, an accurate numerical and experimental analysis has been performed aiming to analyze the aerodynamic performance of a new airfoil.

The analysis has shown that the transition position strongly affects the airfoil performance. With regard to the experimental check, the agreement with numerical results is satisfying. In particular, if the laminar region is accurately predicted, the numerical/experimental comparison is quite good both in terms of local values (pressure and velocity distribution) and global parameters (lift coefficient).

\section{References}

[1] Barlow J. B., Rae W.H., Pope A. "Low Speed Wind Tunnel Testing” John Wiley \&Son, Inc. Third Edition.

[2] Mosharov V., Radchenko V., Fonov S., Luminescent Pressure Sensors in Aerodynamic Experiments, Central Aerohydrodynamic Institute (TsAGI), 1997.

[3] M. Drela, "Newton solution of coupled viscous/inviscid multielement airfoil flows", in AIAA, Fluid Dynamics, Plasma Dynamics and Lasers Conference, AIAA Paper 90-1470, American Institute of Aeronautics and Astronautics (AIAA), Seattle, WA, June 1990.

[4] FLUENT'M 6.1, User's Guide, February 2003. 